\title{
Kinship Asymmetries and the Divided Self
}

\section{Citation}

Haig, David. 2008. Kinship asymmetries and the divided self. Behavioral and Brain Sciences 31(03): 271-272.

\section{Published Version}

doi:10.1017/S0140525X08004329

\section{Permanent link}

http://nrs.harvard.edu/urn-3:HUL.InstRepos:11315418

\section{Terms of Use}

This article was downloaded from Harvard University's DASH repository, and is made available under the terms and conditions applicable to Other Posted Material, as set forth at http:// nrs.harvard.edu/urn-3:HUL.InstRepos:dash.current.terms-of-use\#LAA

\section{Share Your Story}

The Harvard community has made this article openly available.

Please share how this access benefits you. Submit a story.

Accessibility 
growth factor 2 and its receptor, which form a tightly linked physiological unit (Haig \& Graham 1991). In this case, deletion or duplication of either gene produces predictable outcomes of over- or undergrowth. This logic is unlikely to apply, however, in the case of highly variable behavioural phenomena.

The evolutionary forces driving imprinting (Haig \& Westoby 1989) are compounded and exacerbated by those due to sociality (Haig 2000a). In primates, social skills directly influence both survival and reproductive success (Silk 2007). Furthermore, primate social interactions occur primarily with maternal kin (Cheney et al. 1986). In this context, the adaptive resolution of conflict over resource transfer, for maternal and paternal genes, would involve social skills, in order to maximize resource acquisition, minimize individual risk due to conflict, and restrain direct and indirect maternal fitness costs.

Uniparental cell deposition patterns in chimeric mice support this conjecture. Maternally expressed genes contribute primarily to the development of cortical areas mediating complex socio-cognitive skills, such as memory, planning, and language (Allen et al. 1995). The paternal contribution is primary in the mediobasal forebrain and hypothalamus (Keverne et al. 1996). These areas are crucial for adaptive sociality, largely mediated via oxytocin and vasopressin. These hormones reduce social fear, anxiety, and aggression; moderate stress reactivity; facilitate social memory and behavioural flexibility; and coordinate behavioural and emotional responses to social stimuli (Carter \& Altemus 1997; Kirsch et al. 2005; Winslow \& Insel 2004).

If both maternal and paternal genes were selected to maximize fitness in a social milieu, it is likely that imbalance in either direction would result in social deficits. Moreover, different suites of social impairments may legitimately attract the same diagnosis of autism. Relative paternal over-expression due to mutation(s) in maternally expressed genes could produce the cognitive and language deficits characteristic of autism, as suggested by $\mathrm{C} \& \mathrm{~B}$. However, relative maternal over-expression due to mutation(s) in paternally expressed genes could lead to deficits in socio-emotional processing, such as heightened reactivity, anxiety and fear, and stereotypy and rigidity, also characteristic of autism (Corbett et al. 2006; Hollander et al. 2003; Jansen et al. 2003). Indeed, this postulation is consistent with evidence in the literature documenting cortical or neuroendocrine dysfunction in autism, but not necessarily both (Belmonte \& Carper 2006; Chandana et al. 2005; Green et al. 2001; Jacob et al. 2007; Modahl et al. 1998; Wu et al. 2005).

The target article represents an important step in the integration of genomic imprinting and the study of psychopathology. It would, however, be much more compelling and influential if it were more finely parsed with respect to the imprinted genotypes included in it, as well as the behavioural phenotypes it seeks to explain.

\section{ACKNOWLEDGMENTS}

Dr. Goos is supported by a CIHR Postdoctoral Fellowship from the Ontario Women's Health Council. Thanks to Jennifer Crosbie and Russell Schachar for helpful discussions.

\section{Kinship asymmetries and the divided self}

\section{doi:10.1017/S0140525X08004329}

\section{David Haig}

Department of Organismic and Evolutionary Biology, Harvard University, Cambridge, MA 02138.

\section{dhaig@oeb.harvard.edu}

Abstract: Imprinted genes are predicted to affect interactions among relatives. Therefore, variant alleles at imprinted loci are promising candidates for playing a causal role in disorders of social behavior. The effects of imprinted genes evolved in the context of patterns of asymmetric relatedness that existed within social groups of our ancestors.

Genomic imprinting is an expression of an evolutionary conflict, within the genomes of individual organisms, between genes of maternal and paternal origin. It evolves when a genetically determined action enhances the inclusive fitness of madumnal (maternally derived) genes but reduces the inclusive fitness of padumnal (paternally derived) genes, or vice versa. Such situations arise when the expression of a gene in one individual (the actor) has fitness consequences for other individuals who are asymmetric kin of the actor (Haig 1997). (Asymmetric kin are individuals with different probabilities of carrying copies [identical by recent descent] of an actor's madumnal and padumnal alleles. For example, maternal half-sibs are asymmetric kin, related through the actor's mother but not the actor's father, whereas full-sibs are symmetric kin, equally related to the actor via a shared mother and shared father.)

Offspring are usually symmetric kin of parents (a parent's maternal and paternal alleles each has a $50 \%$ chance of being transmitted to offspring) but parents are asymmetric kin of offspring (an offspring's maternal alleles are present in its mother, but the offspring's paternal alleles are absent from its mother). Therefore, an offspring's interactions with its parents are predicted to be more internally conflicted than are the parents' interactions with the offspring. If one assumes that interactions with mothers have been more salient than interactions with fathers, then paternally expressed genes should promote behaviors that engage maternal attention and elicit maternal care, whereas maternally expressed genes should favor greater independence of the child. This is the principal source of asymmetric kinship considered by Crespi \& Badcock $(\mathrm{C} \& \mathrm{~B})$.

Although an offspring usually has an equal chance of inheriting the madumnal or the padumnal allele at a locus in one of its parents, this symmetry breaks down under inbreeding (Wilkins \& Haig 2003). Offspring are asymmetric kin of their mother if their father is asymmetric kin of the mother (and vice versa). For example, a child conceived by father-daughter incest is not only its mother's offspring but also her paternal half-sib. Similarly, the child of a woman who marries her father's sister's son is simultaneously its mother's offspring and her patrilateral first cousin once-removed. In both cases, the child would be more likely to carry copies of its mother's padumnal alleles than copies of her madumnal alleles. In general, a woman's madumnal alleles are predicted to favor greater aversion for sexual relations with patrilineal kin (Haig 1999a).

Sibling rivalry has probably been intensified in recent human evolution because we have shorter interbirth intervals than our closest relatives but longer periods of juvenile dependence (Kennedy 2005). Therefore, sibs will often have competed for resources provided by adults. Full-sibs are symmetric kin, whereas half-sibs are asymmetric kin. The key question for understanding the role of imprinting in sibling rivalry is the relative importance of competition with paternal half-sibs (patrisibs) and maternal half-sibs (matrisibs). If the variance of reproductive success is higher among males than among females, then the population will contain more patrisibs than matrisibs. However, offspring tend to maintain closer relations with mothers than with fathers. Therefore, interactions are likely to have been more intense with matrisibs (the products of female infidelity and partner change) than with patrisibs. My expectation is that madumnally expressed genes will tend to promote cooperative interactions among sibs whereas padumnally expressed genes will favor competitive interactions.

Asymmetries of relatedness are also present in a child's interactions with its extended family. Grandparents, aunts, uncles, cousins, nieces, and nephews are all, with rare exceptions, asymmetric kin. Imprinted behaviors might play a role in broader family relations if a child's interactions were predominantly 
Commentary/Crespi \& Badcock: Psychosis and autism as diametrical disorders of the social brain

with its mother's kin or its father's kin (or if children evolved specific behaviors for interacting with patrilineal and matrilineal kin). Such asymmetries will have been determined by residence patterns and the stability of pair-bonds (Haig 2000a), whether mothers typically resided with their husband's kin or with their own kin, and whether children followed their mother or stayed with their father after divorce.

I foresee two principal challenges to predicting the effects of imprinted genes on social behaviors from evolutionary first principles. The first challenge (discussed above) will be to understand evolutionarily salient patterns of asymmetric relatedness during human ancestry. The second will be to understand the principal psychological dimensions that have differentially affected matrilineal and patrilineal inclusive fitness, given these asymmetries of relatedness. The latter challenge can be illustrated using the example of a curious "gift" of individuals with Prader-Willi syndrome (PWS). Such individuals perform poorly on most cognitive tests but far outperform individuals from the general population on the assembly of jigsaw puzzles (Dykens 2000). PWS is caused by the absence of expression of genes from padumnal chromosome 15q11-13. This suggests that padumnally expressed genes favor relatively greater development of some psychological attribute that has, as a side-effect, poorer performance on fitting together colored pieces of cardboard. Kinship theory predicts that stronger development of this attribute enhanced individual fitness at a cost to mothers or their families (or reduced individual fitness but conferred a benefit on fathers or their families). The challenge is to understand the nature of the attribute that has been subject to natural selection and how expression of this attribute has differentially affected the fitness of mothers and fathers (or their respective kin).

Both challenges remain to be surmounted in the specific cases of autism and schizophrenia. Infantile onset of autism suggests that the relevant genes may have been subject to selection based on their effects on the mother-infant relationship, whereas the older age of onset of schizophrenia hints that the relevant genes may have been subject to selection for their effects in wider kin networks. The greater challenge is understanding what psychological dimensions have been subject to selection and how variation along these dimensions has affected matrilineal and patrilineal fitness. $\mathrm{C} \& \mathrm{~B}$ suggest that autistic features are more pronounced in Angelman syndrome than in Prader-Willi syndrome, whereas Veltman et al. (2005) conclude the opposite. A detailed study of social behavior in these syndromes is a promising way forward.

\section{Are schizophrenics more religious? Do they have more daughters?}

\section{doi:10.1017/S0140525X08004330}

\section{Satoshi Kanazawa}

Interdisciplinary Institute of Management, London School of Economics and Political Science, Houghton Street, London WC2A 2AE; Department of Psychology, University College London, London WC1E 6BT; and Department of Psychology, Birkbeck College, University of London, London WC1E 7HX, United Kingdom.

S.Kanazawa@Ise.ac.uk

http://www.Ise.ac.uk/collections/MES/people/Kanazawa.htm

\begin{abstract}
Combined with recent evolutionary psychological theories, Crespi \& Badcock's (C\&B's) intragenomic conflict theory of the social brain suggests that schizophrenics are more religious, and autistics are less religious, than the normal population. Combined with the generalized Trivers-Willard hypothesis (gTWH), it suggests that schizophrenics have more daughters, and autistics have more sons, than expected.
\end{abstract}

At the end of his book The Essential Difference, which argues that autism and autistic-spectrum syndrome may result from an "extreme male brain," Simon Baron-Cohen asks, "Is there an extreme female brain?” (Baron-Cohen 2003, pp. 170-85). Crespi \& Badcock $(\mathrm{C} \& \mathrm{~B})$ have now answered this question. Contrary to Baron-Cohen, who speculates that, if there is an extreme female brain, it would not be pathological, $C \& B$ suggest, with a considerable amount of supportive evidence, that an extreme female brain, the polar opposite of autism, may amount to schizophrenia and psychotic-spectrum syndrome.

If $\mathrm{C} \& \mathrm{~B}$ are correct, which all available evidence strongly suggests they may be, then their intragenomic conflict theory of the social brain immediately suggests a couple of novel hypotheses. First, recent evolutionary psychological theories (Atran 2002; Boyer 2001; Guthrie 1993; Kirkpatrick 2005; Miller \& Kanazawa 2007, pp. 158-61) suggest that religion is not an adaptation in itself but a by-product of other psychological adaptations, variously named "agency-detection mechanism" or “animistic bias." We may be evolutionarily designed to infer personal and animistic intentions behind natural phenomena, because the consequence of erroneously overinferring intentions - being paranoid about perfectly natural phenomena - is much less costly in evolutionary terms than the consequence of erroneously underinferring intentions - being killed by predators and enemies when we least expect them (Haselton \& Nettle 2006). We may therefore be designed to be paranoid because it can potentially save our lives, and we may be religious because we are paranoid and see the "hands of God" behind completely natural phenomena.

If this view is correct, then C\&B's theory suggests that schizophrenics, who are hypermentalistic and are more "paranoid," may be predisposed to be more religious, more prone to see the hands of God behind natural phenomena, just as some (McNamara 2001; Miller \& Kanazawa 2007, p. 206, n13) suggest that autistics should be less religious because of their hypomentalism (absence of theory of mind). C\&B identify "overestimation of meaningfulness of naturally occurring coincidences" (sect. 6.1.3, para. 2) as one of the symptoms of schizophrenia.

In virtually all nations of the world, women are more religious than men. While Alan S. Miller and I (Miller \& Kanazawa 2007, pp. 161-65; cf. Miller \& Stark 2002) explain this in terms of women's greater tendency toward risk aversion, C\&B's theory suggests another explanation: If religion is mentalizing natural phenomena, and if the female brain tends toward mentalizing, then women should naturally be more religious.

Second, in an entirely different area, the generalized TriversWillard hypothesis (gTWH) (Kanazawa 2005) proposes that parents who possess any heritable trait which increases male reproductive success at a greater rate (or decreases male reproductive success at a smaller rate) than female reproductive success in a given environment, will have higher-than-expected offspring sex ratios (more sons). Conversely, parents who possess any heritable trait which increases female reproductive success at a greater rate (or decreases female reproductive success at a smaller rate) than male reproductive success in a given environment, will have lower-than-expected offspring sex ratios (more daughters). Because body size and tendency toward violence are distinct advantages in male intrasexual competition for mates and status, big and tall parents are more likely to have sons (Kanazawa 2005; 2007b), and violent men are more likely to have sons (Kanazawa 2006). Because physical attractiveness, while an advantage for both men and women, is even a greater advantage for women than for men, more beautiful parents are more likely to have daughters (Kanazawa 2007a). Because language and communication are more important to women, and thus language impairment is relatively more costly for them, mothers (though not fathers) with a developmental language impairment have more sons than daughters (Tallal et al. 1989).

I have applied the gTWH to brain types and shown that those with "strong male brains," such as engineers, mathematicians, 